\title{
Regeneration of Parietal Cells at the Healing Process of Acetic Acid-induced Gastric Ulcers in Rats - Evaluation of the Quality of Ulcer Healing by the Administration of Various Agents-
}

\author{
Katsuya Nakano, Hideyuki Konishi, Masao Noda, Hitomi Sato ${ }^{*}$ **, \\ Tadashi Kodama, Kei Kashima ${ }^{*}$ and Takanori Hattori** \\ *Third Department of Internal Medicine, Kyoto Prefectural University of Medicine, Kyoto 602, \\ ${ }^{* *}$ Department of Pathology, Shiga University of Medical Science, Shiga 520
}

Key words: Experimental ulcer, Parietal cell, Quality of ulcer healing

\section{Introduction}

There are various methods that induce gastric ulcers in experimental animals, but it is difficult to evaluate them as identical to those of human. In experimental animals, it is difficult to make chronic gastritis, but in humans, the natural history of ulcer healing is certainly influenced by gastritis. On the other hands, as human gastric ulcers recur easily, the quality of ulcer healing has been discussed from many points of view. In this study, we used an experimental ulcer model and investigated the regeneration of parietal cells to assess the quality of ulcer healing after administration of various agents.

\section{Materials and Methods}

Male Wistar rats, weighting $200-250 \mathrm{~g}$ were used. Acetic acid ulcers were induced on the anterior wall of the corpus region of rat stomachs according to the method of Okabe, et al [2]. Then various agents, including epidermal growth factor, famotidine, omeplazole, teprenone and indomethacin were administered every day from 2 months after the induction of ulcers. As a control group, only gum Arabic solution was administered. We sacrificed rats every 3 months after the administration of these agents. After sacrifice, the resected stomach was incised along the greater curvature and cut into two portions along the long axis at the center of ulcer. The one for immunoflourescent study was embedded in OCT compound and cooled to the temperature of liquid nitrogen and the other for HE staining was fixed in 10\% formalin and embedded in paraffin.

Presented in part at the workshop 2 on Histochemistry of Gastric Ulcers and Related Diseases, July 14, 1994 as part of the program at the 4th Joint Meeting of the Japan Society of Histochemistry and Cytochemistry and the Histochemical Society, held in Maui, Hawaii, July 13 to 16,1994 .

\section{Evaluation of regeneration of parietal cells}

Frozen sections were cut at 5 um, collected on coated slides, fixed in cold acetone (10 min.) and air-dried. As for immunofluorescent study, we used mouse serum including anti-parietal cell antibody which was obtained from autoimmune gastritis mice [1]. The antigen/antibody complexes were visualized by a fluorescein isothiocyanate (FITC). Then we studied the number of parietal cells by ocular eyegrid.

\section{Electron microscopic study}

After sacrifice, the specimens for electron microscopy were taken from regenerated mucosa. Small pieces were fixed in $2.5 \%$ glutaraldehyde, postfixed in $1 \%$ osmium tetroxide and embedded in Epon812. Ultrathin sections were cut and were double-stained by uranyl acetate and lead citrate. Then we examined the occurence of parietal cells with electron microscope.

\section{Results}

Normal healing process of acetic acid-induced gastric ulcers in rats

At 1 month after the operation, new glands were formed by budding of cystic glandular tubules with subsequent differentiation to a certain mucous gland cell, and the regenerative mucosa covered the ulcerated region (Fig. 1A). At 3 months after the operation, the height of these mucous glands were still lower than that of the normal fundic mucosa and there were no proper gastric glands (Fig. 1B). At 6 months after the operation, immature parietal cells began to appear, and it was not until 9 months after the operation, when thickness of the mucosa became as thick as that of the normal fundic mucosa, that many parietal cells were identified (Fig. 1C.). 

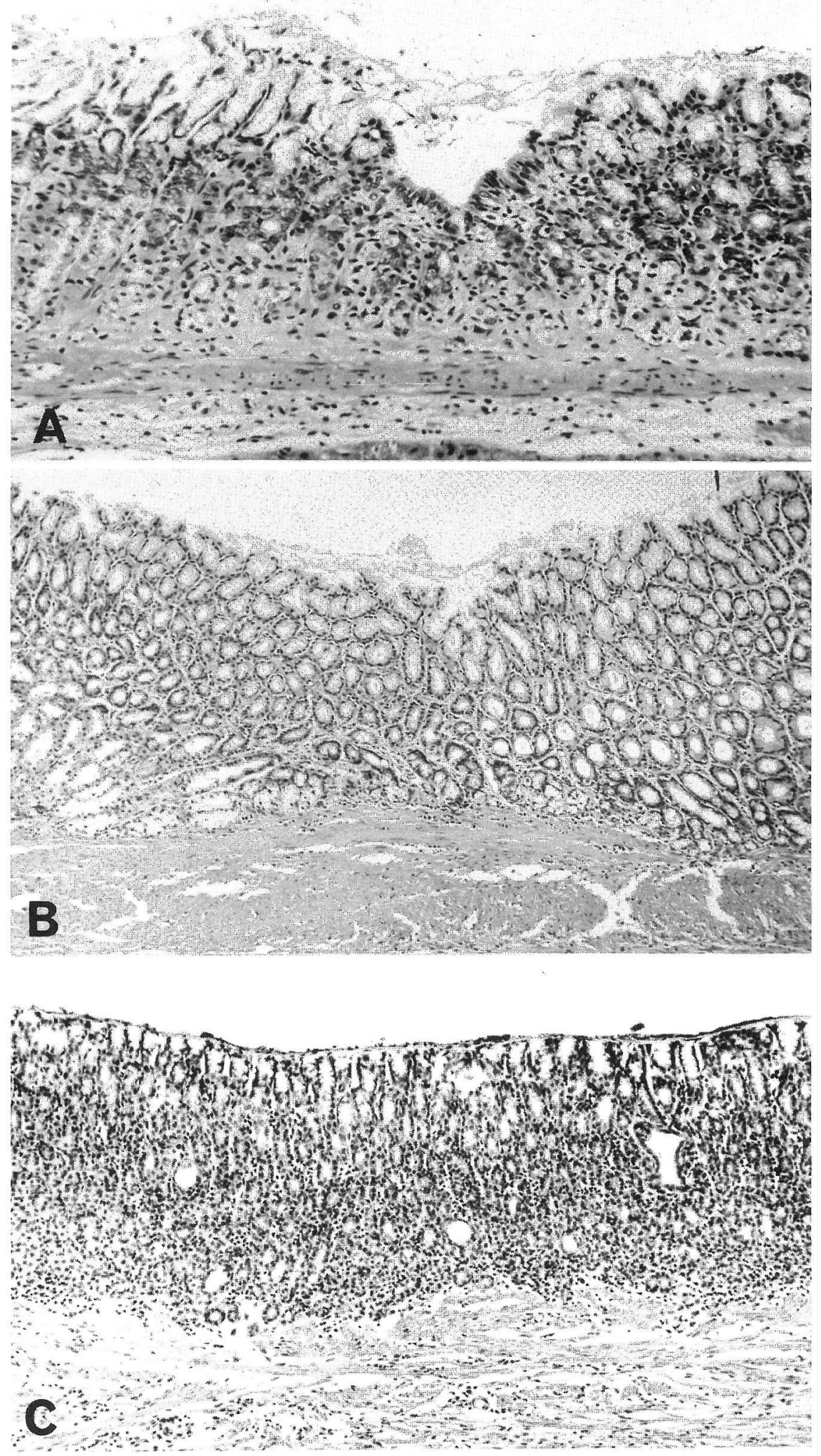

Fig. 1. Normal healing process of acetic acid-induced gastric ulcer in rat. A: At 1 month. B: At 3 months. C: At 9 months. 


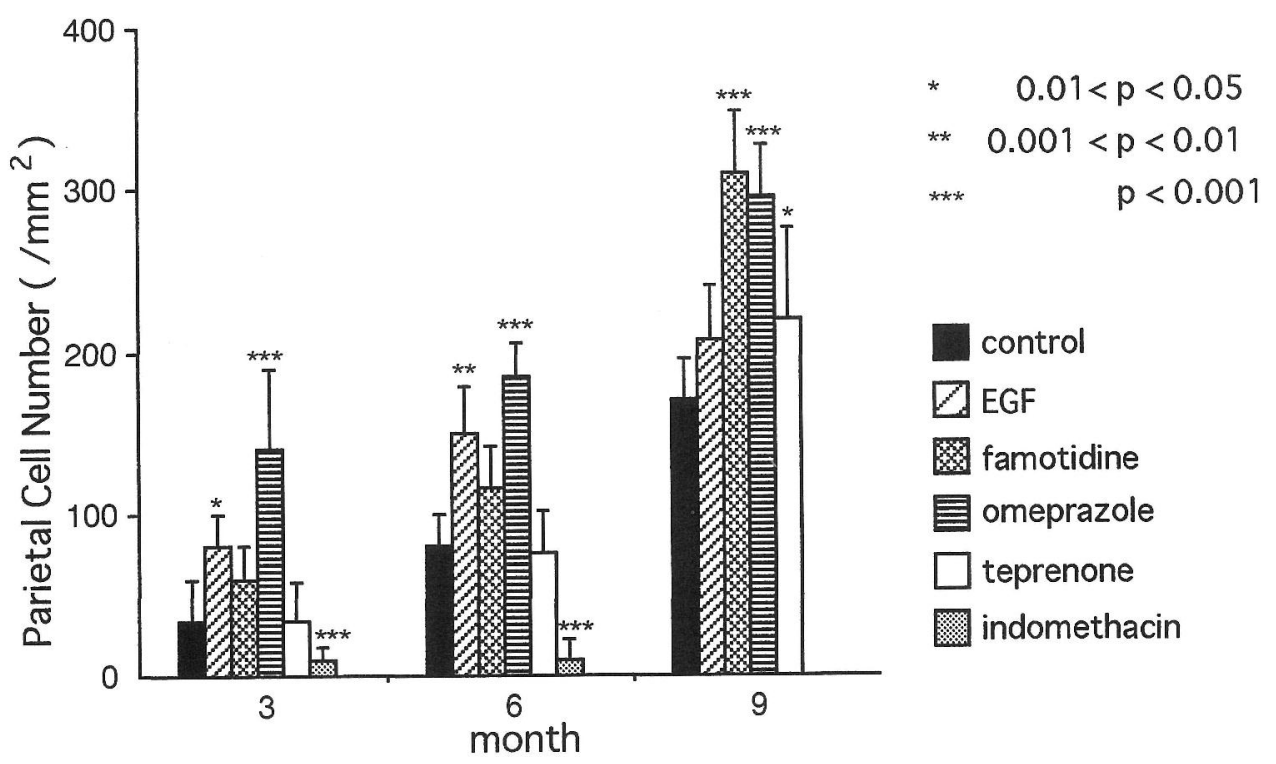

Fig. 2. Changes in parietal cell number at acetic acid-induced gastric ulcer during the administration of various agents.
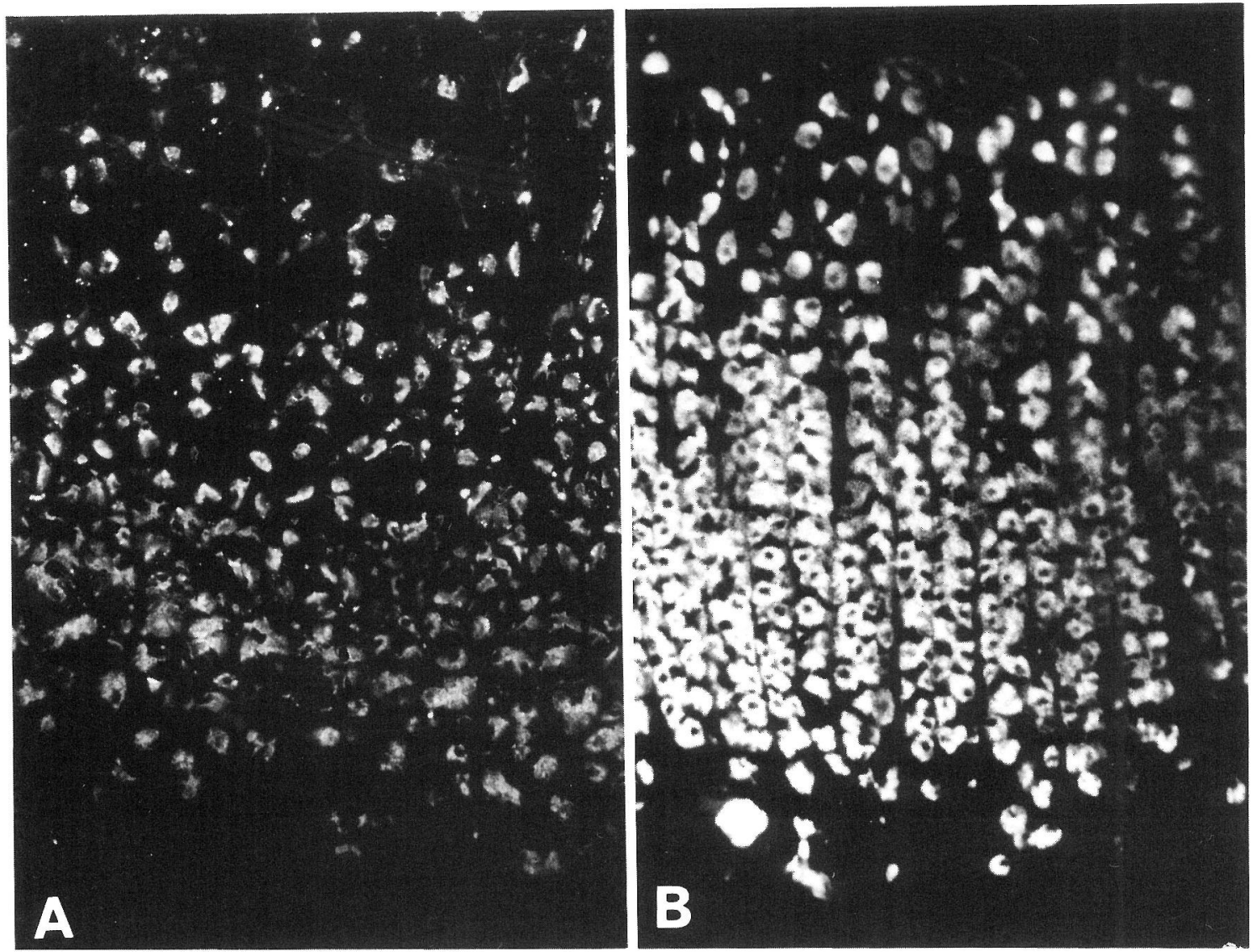

Fig. 3. Immunofluorescence staining of parietal cell at 9 months. A: Control. B: After the administration of omeplazole. 


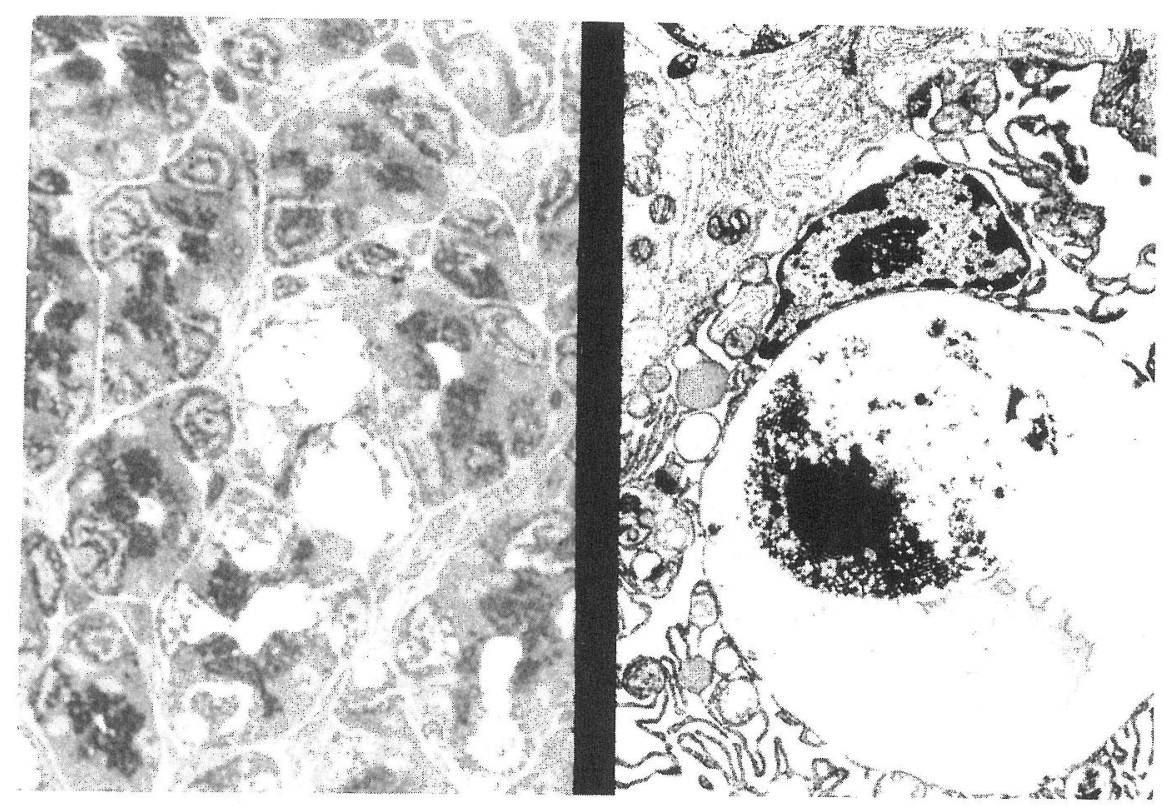

Fig. 4. Electron microscopic study of the necrotic parietal cell.

\section{Effects of various drugs upon the regeneration of parietal} cells (Fig. 2)

At 3 months after the administration of mucosal protectant and acid secretion inhibitors, immature parietal cells began to appear, and at 6 and 9 months after the administration, more parietal cells regenerated significantly, compared with those of the control group (Fig. 3A, B).

Omeprazole has accelerated the regeneration of parietal cells during the examination period. Famotidine and epidermal growth factor accelerated it at the early phase and teprenone accelerated it only at the late phase. For the rats which were administered indomethacin, ulcer remained to be open or only covered with mucous glands. So, in this group, no definite parietal cells appeared during the experimental period.

In the group of omeprazole administration, we could find some necrotic parietal cells probably due to hyperstimulation by proton pump inhibition with electron microscopic study. (Fig. 4)

\section{Conclusions}

In evaluating the quality of ulcer healing and the effectiveness of agents on the healing process of ulcer healing, it seems to be useful to investigate the regeneration of parietal cells at experimental ulcers.

\section{References}

1. Fukuma, K., Sakaguchi, S., Kuribayashi, K., Chen, WL., Morishita, R., Sekita, K., Uchino, H. and Masuda T.: Immunologic and clinical studies on murine experimental autoimmune gastritis induced by neonatal thymectomy. Gastroenterol. 94; 274-283, 1988.

2. Okabe, S., Roth, JLA. and Pfeiffer CJ.: A method for experimental penetrating gastric and duodenal ulcers in rats. Observations on normal healing. Amer. J. Dig. Dis. 16; 277284, 1971. 